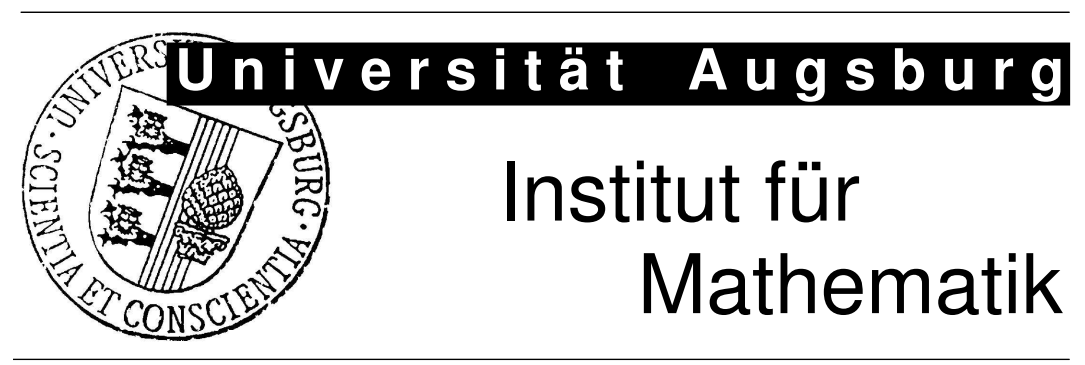

Ronald H.W. Hoppe, Guido Kanschat, Tim Warburton

Convergence Analysis of an Adaptive Interior Penalty Discontinuous Galerkin Method 


\section{Impressum:}

Herausgeber:

Institut für Mathematik

Universität Augsburg

86135 Augsburg

http://www . math. uni-augsburg.de/forschung/preprint/

ViSdP:

Ronald H.W. Hoppe

Institut für Mathematik

Universität Augsburg

86135 Augsburg

Preprint: Sämtliche Rechte verbleiben den Autoren (C) 2007 


\title{
CONVERGENCE ANALYSIS OF AN ADAPTIVE INTERIOR PENALTY DISCONTINUOUS GALERKIN METHOD
}

\author{
R. H. W. HOPPE* ${ }^{*}$, G. KANSCHAT ${ }^{\ddagger}$, AND T. WARBURTON $^{\S}$
}

\begin{abstract}
We study the convergence of an adaptive Interior Penalty Discontinuous Galerkin (IPDG) method for a 2D model second order elliptic boundary value problem. Based on a residualtype a posteriori error estimator, we prove that after each refinement step of the adaptive scheme we achieve a guaranteed reduction of the global discretization error in the mesh dependent energy norm associated with the IPDG method. In contrast to recent work on adaptive IPDG methods [29], the convergence analysis does not require multiple interior nodes for refined elements of the triangulation and thus leads to a more efficient adaptive scheme. In fact, it will be shown that bisection of elements is sufficient. The main ingredients of the proof of the error reduction property are the reliability and a perturbed discrete local efficiency of the estimator, a bulk criterion that takes care of a proper selection of edges and elements for refinement, and a perturbed Galerkin orthogonality property with respect to the energy inner product. Results of numerical experiments are given to illustrate the performance of the adaptive method.
\end{abstract}

1. Introduction. During the past decade, discontinuous Galerkin (DG) methods have emerged as a powerful algorithmic tool in the numerical solution of boundary and initial-boundary value problems for partial differential equations (PDE) (cf., e.g., $[15,16]$ and the references therein). For second order elliptic problems, one may distinguish between primal schemes and mixed methods. Primal schemes rely on augmenting the elliptic operator by an appropriate penalization of the discontinuous nodal shape functions. On the other hand, in mixed methods the second order PDE is reformulated as a system of first order PDEs for which suitable numerical fluxes are designed. The most prominent primal schemes are Interior Penalty Discontinuous Galerkin (IPDG) methods, whereas a widely used class of mixed techniques is given by the Local Discontinuous Galerkin (LDG) methods. Both IPDG and LDG methods have been intensively studied with regard to an a priori error analysis in terms of error estimates for the global discretization error (see, e.g., [4, 12, 22, 27]).

The a posteriori analysis of finite element methods (FEM) is in some state of maturity as documented by a series of monographs that have been published in recent years $[1,5,6,19,33,38]$. As far as DG methods are concerned, a posteriori error estimators have been developed and analyzed for elliptic problems in $H^{1}$ in $[7,26,28$, $34,35]$, for elliptic problems in $H(\mathrm{curl})$ in [23, 24], and for the Stokes problem in [25].

In this paper, we will be concerned with a convergence analysis of an adaptive IPDG method in the sense that for a 2D second order elliptic model problem we will prove guaranteed error reduction with respect to the problem related mesh dependent energy norm. We note that for standard conforming P1 approximations of elliptic problems the convergence analysis of adaptive finite element methods (AFEM) has been initiated in [18] and further studied in [30,31,32], whereas the issue of optimal order of convergence has been addressed in [8] and [37]. Non-standard finite element techniques such as mixed and nonconforming methods and edge element discretizations of Maxwell's equations have been recently investigated in $[9,10,11]$. In

*Dept. of Math., Univ. of Houston, Houston, TX 77204-3008, U.S.A. Supported by NSF grants DMS-0511611 and DMS-0707602

$\dagger$ Inst. of Math., Univ. of Augsburg, D-86159 Augsburg, Germany

$\ddagger$ Dept. of Math., Texas A\&M University, College Station, TX 77843-3368, U.S.A. Supported by NSF grant DMS-0713829

${ }^{\S}$ CAAM, Rice Univ., Houston, TX 77005-1892, U.S.A. Supported by grants NSF-DMS-0512673 and FA9550-05-1-0473 
the recent paper [29], a convergence analysis of symmetric IPDG methods has been provided. In contrast to [29], our analysis does not require multiple interior nodes for refined elements of the triangulation. In fact, we show that it suffices to refine by bisection. Hence, we end up with a computationally more efficient adaptive scheme.

The paper is organized as follows: In section 2, we briefly introduce the IPDG. Section 3 describes the adaptive loop consisting of the basic steps SOLVE, ESTIMATE, MARK and REFINE and states the main convergence result. Section 4 recalls the reliability of the estimator from [28] and establishes a perturbed discrete local efficiency, whereas section 5 is devoted to the proof of the error reduction property. Finally, Section 6 contains a documentation of the results of numerical experiments that illustrate the performance of the AIPDG.

2. The interior penalty discontinuous Galerkin method. We assume $\Omega \subset$ $\mathbb{R}^{2}$ to be a bounded, polygonal domain with boundary $\Gamma=\partial \Omega, \Gamma=\Gamma_{D} \cup \Gamma_{N}, \Gamma_{D} \cap$ $\Gamma_{N}=\emptyset$. We adopt standard notation from Sobolev space theory and refer to $(\cdot, \cdot)_{k, D}$ and $\|\cdot\|_{k, D}, k \in \mathbb{N}_{0}, D \subseteq \Omega$, as the $H^{k}(D)$-inner product and associated norm, respectively.

As a model problem, for given $f \in L^{2}(\Omega), u^{D} \in H^{1 / 2}\left(\Gamma_{D}\right), u^{N} \in L^{2}\left(\Gamma_{N}\right)$ we consider Poisson's equation with inhomogeneous Dirichlet and Neumann boundary data

$$
\begin{aligned}
-\Delta u=f & \text { in } \Omega, \\
u=u^{D} & \text { on } \Gamma_{D}, \\
\partial_{n_{\Gamma_{N}}} u=u^{N} & \text { on } \Gamma_{N},
\end{aligned}
$$

whose variational formulation amounts to the computation of a solution $u \in V:=$ $\left\{v \in H^{1}(\Omega)|v|_{\Gamma_{D}}=u^{D}\right\}$ such that

$$
a(u, v)=(f, v)_{\Omega}+\left\langle u^{N}, v\right\rangle_{\Gamma_{N}} \quad, \quad v \in H_{0, \Gamma_{D}}^{1}(\Omega),
$$

where $a(u, v):=\int_{\Omega} \nabla u \cdot \nabla v d x$.

For the discontinuous Galerkin approximation of $(2.2)$ we further assume that $\mathcal{T}_{H}(\Omega)$ is a simplicial triangulation of $\Omega$ which aligns with $\Gamma_{D}, \Gamma_{N}$ on the boundary $\Gamma$. For $D \subseteq \bar{\Omega}$, we denote by $|D|$ the volume of $D$ and by $\Pi_{p}(D), p \in \mathbb{N}_{0}$, the linear space of polynomials of degree $p$ on $D$, and we refer to $\mathcal{N}_{H}(D), \mathcal{E}_{H}(D)$ and $\mathcal{T}_{H}(D)$ as the sets of vertices, edges and elements in $D$. For $T \in \mathcal{T}_{H}(\Omega), h_{T}$ stands for the diameter of $T$, whereas for $E \in \mathcal{E}_{H}(\bar{\Omega})$ we denote by $h_{E}$ the length of $E$. Moreover, for an interior edge $E \in \mathcal{E}_{H}(\Omega)$ such that $E=T_{+} \cap T_{-}, T_{ \pm} \in \mathcal{T}_{H}(\Omega)$, we refer to $\omega_{E}:=T_{+} \cup T_{-}$as the patch formed by the union of the elements sharing $E$ as a common edge. Finally, for a function $g \in L^{2}(D), D \subset \bar{\Omega}$, the quantity $\hat{g}_{D}$ stands for the integral mean of $g$ with respect to $D$, i.e., $\hat{g}_{D}:=|D|^{-1} \int_{D} g d x$.

We define the product space $V_{H}:=\prod_{T \in \mathcal{T}_{H}(\Omega)} \Pi_{p}(T), p \in \mathbb{N}$, and introduce the bilinear form $a_{H}(\cdot, \cdot): V_{H} \times V_{H} \rightarrow \mathbb{R}$ according to

$$
\begin{aligned}
a_{H}\left(u_{H}, v_{H}\right): & =\sum_{T \in \mathcal{T}_{H}(\Omega)}\left(\nabla u_{H}, \nabla v_{H}\right)_{T} \\
- & \sum_{E \in \mathcal{E}_{H}\left(\Omega \cup \Gamma_{D}\right)}\left\{\left(\partial_{n_{E}}\left\{u_{H}\right\},\left[v_{H}\right]\right)_{E}+\left(\left[u_{H}\right]_{E}, \partial_{n_{E}}\left\{v_{H}\right\}\right)_{E}\right\} \\
& +\sum_{E \in \mathcal{E}_{H}\left(\Omega \cup \Gamma_{D}\right)} \frac{\alpha}{h_{E}}\left(\left[u_{H}\right]_{E},\left[v_{H}\right]_{E}\right)_{E},
\end{aligned}
$$


where the normal vector on $E$ points from $T_{+}$to $T_{-}$and with $v_{H}^{ \pm}:=\left.v_{H}\right|_{T_{ \pm}}$on $E$

$$
\begin{aligned}
{\left[v_{H}\right]_{E} } & :=v_{H}^{+}-v_{H}^{-} & & E \in \mathcal{E}_{H}(\Omega), \\
{\left[v_{H}\right]_{E} } & :=\left.v_{H}\right|_{E}, & & E \in \mathcal{E}_{H}(\Gamma), \\
\left\{v_{H}\right\}_{E} & :=\frac{1}{2}\left(v_{H}^{+}+v_{H}^{-}\right), & & E \in \mathcal{E}_{H}(\Omega), \\
\left\{v_{H}\right\}_{E} & :=\left.v_{H}\right|_{E}, & & E \in \mathcal{E}_{H}(\Gamma) .
\end{aligned}
$$

The penalty parameter $\alpha>0$ has to be chosen properly. Its lower limit can be computed for each cell by an inverse estimate on each cell. The correct formulation would therefore involve the diameter of the cell perpendicular to $E$ instead of $h_{E}$; for shape regular meshes, we replace it by $h_{E}$ to simplify the presentation below. Ideally, $\alpha$ on each edge $E$ should be chosen separately as the sum of the values obtained from both adjacent cells. We remark here, that the arguments below hold for such local stabilization parameters as well.

Then, the (symmetric) interior penalty method amounts to the computation of $u_{H} \in V_{H}$ such that

$$
a_{H}\left(u_{H}, v_{H}\right)=\ell\left(v_{H}\right) \quad, \quad v_{H} \in V_{H} .
$$

where

$$
\ell\left(v_{H}\right):=\left(f, v_{H}\right)_{\Omega}+\left(u^{N}, v_{H}\right)_{\Gamma_{N}}-\sum_{E \subset \Gamma_{D}}\left(u^{D}, \partial_{n} v_{H}-\frac{\alpha}{h_{E}} v_{H}\right)_{E} .
$$

On $V_{H}$, we introduce the mesh-dependent $H^{1}$-norm defined by

$$
\left\|v_{H}\right\|_{1, H, \Omega}^{2}:=\sum_{T \in \mathcal{T}_{H}(\Omega)}\left\|\nabla v_{H}\right\|_{T}^{2}+\sum_{E \in \mathcal{E}_{H}(\bar{\Omega})}\left(h_{E}\left\|\partial_{n_{E}}\left\{v_{H}\right\}\right\|_{E}^{2}+\frac{\alpha}{h_{E}}\left\|\left[v_{H}\right]\right\|_{E}^{2}\right) .
$$

As has been shown in [28], the bilinear form $a_{H}(\cdot, \cdot)$ is bounded

$$
\left|a_{H}\left(u_{H}, v_{H}\right)\right| \leq(1+\alpha)\left\|u_{H}\right\|_{1, H, \Omega}\left\|v_{H}\right\|_{1, H, \Omega}, \quad u_{H}, v_{H} \in V_{H}
$$

and for sufficiently large $\alpha$ coercive with respect to the $\|\cdot\|_{1, H, \Omega}$-norm, i.e., there exist positive constants $\alpha_{\text {min }}$ and $\gamma$ such that for $\alpha \geq \alpha_{\text {min }}$

$$
a_{H}\left(v_{H}, v_{H}\right) \mid \geq \gamma\left\|v_{H}\right\|_{1, H, \Omega}^{2} \quad, \quad v_{H} \in V_{H} .
$$

It follows from (2.7) and (2.8) that for $\alpha \geq \alpha_{\min }$ the IPDG (2.4) admits a unique solution $u_{H} \in V_{H}$. Moreover, for such $\alpha$ the mesh-dependent energy norm

$$
\left\|v_{H}\right\|_{H, \Omega}:=a_{H}\left(v_{H}, v_{H}\right)^{1 / 2} \quad, \quad v_{H} \in V_{H}
$$

is equivalent to the $\|\cdot\|_{1, H, \Omega}$-norm

$$
\gamma\left\|v_{H}\right\|_{1, H, \Omega}^{2} \leq\left\|v_{H}\right\|_{H, \Omega}^{2} \leq(1+\alpha)\left\|v_{H}\right\|_{1, H, \Omega}^{2} \quad, \quad v_{H} \in V_{H} .
$$

For a subset $D_{H} \subset \mathcal{T}_{H}(\Omega)$ of the triangulation, $\|\cdot\|_{1, H, D_{H}}$ and $\||\cdot|\|_{H, D_{H}}$ are defined analogously. 
3. The adaptive loop and the main convergence result. An adaptive finite element method for the IPDG (2.4) consists of successive loops of the following sequence

$$
\mathrm{SOLVE} \rightarrow \text { ESTIMATE } \rightarrow \text { MARK } \rightarrow \text { REFINE }
$$

Here, SOLVE stands for the numerical solution of (2.4) with respect to the given triangulation $\mathcal{T}_{H}(\Omega)$. We remark that for this purpose efficient preconditioned iterative solvers have been developed, analyzed and implemented (cf., e.g., [2, 20, 21, 26]).

The following residual-type a posteriori error estimator $\eta_{H}$ has been introduced and analyzed in [28]:

$$
\eta_{H}^{2}:=\sum_{T \in \mathcal{T}_{H}(\Omega)} \eta_{T}^{2}+\sum_{E \in \mathcal{E}_{H}(\Omega)} \eta_{E}^{2}
$$

Here, $\eta_{T}$ stands for the element residual

$$
\eta_{T}:=h_{T}\left\|f+\Delta u_{H}\right\|_{T} \quad, \quad T \in \mathcal{T}_{H}(\Omega) .
$$

On the other hand, $\eta_{E}$ summarizes the edge residuals

$$
\eta_{E}^{2}:=\eta_{E, 1}^{2}+\eta_{E, 2}^{2}+\eta_{E, N}^{2}+\eta_{E, D}^{2},
$$

given by

$$
\begin{aligned}
\eta_{E, 1}:=h_{E}^{1 / 2}\left\|\partial_{n_{E}}\left[u_{H}\right]\right\|_{E}, & & E \in \mathcal{E}_{H}(\Omega), \\
\eta_{E, 2}:=\sqrt{\frac{\alpha}{h_{E}}}\left\|\left[u_{H}\right]\right\|_{E}, & & E \in \mathcal{E}_{H}(\Omega), \\
\eta_{E, N}:=h_{E}^{1 / 2}\left\|u^{N}-\partial_{n_{E}} u_{H}\right\|_{E}, & & E \in \mathcal{E}_{H}\left(\Gamma_{N}\right), \\
\eta_{E, D}:=\sqrt{\frac{\alpha}{h_{E}}}\left\|u^{D}-u_{H}\right\|_{E}, & & E \in \mathcal{E}_{H}\left(\Gamma_{D}\right) .
\end{aligned}
$$

The convergence analysis further invokes the data oscillations

$$
\operatorname{osc}_{H}^{2}:=\operatorname{osc}_{H}^{2}(f)+\operatorname{osc}_{H}^{2}\left(u^{D}\right)+\operatorname{osc}_{H}^{2}\left(u^{N}\right),
$$

where

$$
\begin{aligned}
\operatorname{osc}_{H}^{2}(f) & :=\sum_{T \in \mathcal{T}_{H}(\Omega)} \operatorname{osc}_{T}^{2}(f), \\
\operatorname{osc}_{T}(f) & :=h_{T}\left\|f-\hat{f}_{T}\right\|_{T}, \\
\operatorname{osc}_{H}^{2}\left(u^{D}\right) & :=\sum_{E \in \mathcal{E}_{H}\left(\Gamma_{D}\right)} \operatorname{osc}_{E}^{2}\left(u^{D}\right), \\
\operatorname{osc}_{E}\left(u^{D}\right) & :=\sqrt{\frac{\alpha}{h_{E}}}\left\|u^{D}-\hat{u}_{E}^{D}\right\|_{E}, \\
\operatorname{osc}_{H}^{2}\left(u^{N}\right) & :=\sum_{E \in \mathcal{E}_{H}\left(\Gamma_{N}\right)} \operatorname{osc}_{E}^{2}\left(u^{N}\right), \\
\operatorname{osc}_{E}\left(u^{N}\right) & :=h_{E}^{1 / 2}\left\|u^{N}-\hat{u}_{E}^{N}\right\|_{E} .
\end{aligned}
$$


In the Step 'MARK' of the adaptive loop, given a universal constant $\Theta$, we choose subsets $\mathcal{M}_{T} \subset \mathcal{T}_{H}(\Omega)$ and $\mathcal{M}_{E} \subset \mathcal{E}_{H}(\bar{\Omega})$ such that the following bulk criterion is satisfied

$$
\begin{aligned}
& \Theta \sum_{T \in \mathcal{T}_{H}(\Omega)} \eta_{T}^{2} \leq \sum_{T \in \mathcal{M}_{T}} \eta_{T}^{2}, \\
& \Theta \sum_{E \in \mathcal{E}_{H}(\bar{\Omega})} \eta_{E}^{2} \leq \sum_{E \in \mathcal{M}_{E}} \eta_{E}^{2} .
\end{aligned}
$$

The bulk criterion can be realized by a greedy algorithm.

As far as the data oscillations are concerned, for simplicity we assume that the sets $\mathcal{M}_{T}$ and $\mathcal{M}_{E}$ selected by (3.8a) and (3.8b) are already rich enough such that there exists a constant $0 \leq \rho_{2}<1$ such that

$$
\operatorname{osc}_{h}^{2} \leq \rho_{2} \operatorname{osc}_{H}^{2} .
$$

We note that the data oscillations may be included in the bulk criterion as well to guarantee (3.9). We refer to [31, 32] for details.

The refinement strategy in the final step REFINE of the adaptive loop is as follows: If an element $T \in \mathcal{T}_{H}(\Omega)$ has been marked for refinement, it will be refined by longest edge bisection. If an edge $E \in \mathcal{E}_{H}(\Omega), E=T^{+} \cap T^{-}$, (resp. $\left.E \in \mathcal{T}_{H}(\Gamma), E=\partial T \cap \Gamma\right)$ has been marked, the triangles $T^{ \pm}$(resp. the triangle $T$ ) will be refined by bisection. We note that this refinement leads to a much more efficient adaptive scheme compared to [29] where the refinement of a triangle requires multiple interior nodes based on subsequent regular refinements.

The main result of this paper is a guaranteed error reduction of the global discretization error measured in the mesh dependent energy norm associated with the IPDG method.

THEOREM 3.1. Let $u \in V$ be the solution of (2.2) and suppose that $u_{H} \in V_{H}$ and $u_{h} \in V_{h}$ are the solutions of IPDG (2.4) with respect to the triangulation $\mathcal{T}_{H}(\Omega)$ and the adaptively refined triangulation $\mathcal{T}_{h}(\Omega)$ generated according to the refinement rules described before. Assume that (3.9) holds true. Then, for sufficiently large penalization parameter $\alpha$ there exist positive constants $\varrho_{1}<1$ and $C$ which only depend on $\alpha, \Theta$ and the shape regularity of the triangulations such that for $e_{H}:=u-u_{H}$ and $e_{h}:=u-u_{H}$ there holds

$$
\left(\begin{array}{c}
a_{h}\left(e_{h}, e_{h}\right) \\
\operatorname{osc}_{h}^{2}
\end{array}\right) \leq\left(\begin{array}{cc}
\rho_{1} & C \\
0 & \rho_{2}
\end{array}\right)\left(\begin{array}{c}
a_{H}\left(e_{H}, e_{H}\right) \\
\operatorname{osc}_{H}^{2}
\end{array}\right) .
$$

The proof of Theorem 3.1 will be given in section 5 based on the reliability and a perturbed discrete local efficiency of the estimator (3.2) which will be studied in the following section.

4. Reliability and perturbed discrete local efficiency. The reliability of the residual type a posteriori error estimator (3.2) has been established in [28] using standard techniques from AFEM [38]. Here, we prove that it is also locally efficient in a relaxed way. We will derive the main lemmas for the case of newest edge bisection [13, $14,36]$.

THEOREM 4.1. Let $u \in V$ and $u_{H} \in V_{H}$ be the solution of (2.2) and its IPDG approximation (2.4) and let $\eta_{H}$ and $\operatorname{osc}_{H}$ be the residual error estimator and the data 
oscillations as given by (3.2) and (3.6), respectively. Then, for $e_{H}:=u-u_{H}$ there holds

$$
a_{H}\left(e_{H}, e_{H}\right) \lesssim \eta_{H}^{2}+\operatorname{osc}_{H}^{2} .
$$

Discrete local efficiency means that up to data oscillations the local contributions of the estimator can be bounded from above by the energy norm of the difference between the fine mesh and coarse mesh approximations on a refined triangle and the patch $\omega_{E}$ associated with a refined edge, respectively [18, 31]. In the framework of the IPDG approximations under consideration, we can only prove a perturbed discrete local efficiency in the sense that the upper bounds involve additional quantities in terms of the fine mesh approximation. In particular, the following result holds true.

TheOREM 4.2. Let $u \in V$ and $u_{H} \in V_{H}, u_{h} \in V_{h}$ be the solution of (2.2) and its IPDG approximations $(2.4)$ with respect to $\mathcal{T}_{H}(\Omega)$ and $\mathcal{T}_{h}(\Omega)$, respectively. Moreover, let $\eta_{H}$ and $\operatorname{osc}_{H}$ be the residual error estimator (3.2) and the data oscillations (3.6). Then, there holds

$$
\begin{aligned}
\sum_{T \in \mathcal{M}_{T}} \eta_{T}^{2} & +\sum_{E \in \mathcal{M}_{E}} \eta_{E}^{2} \lesssim a_{h}\left(u_{h}-u_{H}, u_{h}-u_{H}\right)+ \\
& +\sum_{E \in \mathcal{M}_{E} \cap \mathcal{E}_{h}(\Omega)} \frac{\alpha}{h_{E}}\left\|\left[u_{h}\right]\right\|_{E}^{2}+\sum_{E \in \mathcal{M}_{E} \cap \mathcal{E}_{h}\left(\Gamma_{D}\right)} \frac{\alpha}{h_{E}}\left\|u^{D}-u_{h}\right\|_{E}^{2}+\operatorname{osc}_{H}^{2}
\end{aligned}
$$

Proof. The proof of (4.2) follows by collecting the estimates from the subsequent series of lemmas.

LEMma 4.3. Let $T \in \mathcal{T}_{H}(\Omega)$ be a refined triangle such that $T=T_{1} \cup T_{2}, T_{i} \in$ $\mathcal{T}_{h}(\Omega)$. Then, there holds

$$
\begin{aligned}
&\left.h_{T}^{2}\left\|f+\Delta u_{H}\right\|_{T}^{2} \lesssim a_{h}\right|_{T}\left(u_{h}-u_{H}, u_{h}-u_{H}\right)+\operatorname{osc}_{T}^{2}(f) \\
&+\sum_{E \in \mathcal{E}_{H}(\partial T \cap \Omega)} \eta_{E, 2}^{2}+\sum_{E \in \mathcal{E}_{H}\left(\partial T \cap \Gamma_{D}\right)} \eta_{E, D}^{2}+\sum_{E \in \mathcal{E}_{H}\left(\partial T \cap \Gamma_{N}\right)} \operatorname{osc}_{E}^{2}\left(u^{N}\right) .
\end{aligned}
$$

Proof. We denote by $C R_{p}\left(\Omega ; \mathcal{T}_{h}(\Omega)\right), p \in \mathbb{N}$, the nonconforming Crouzeix-Raviart finite element space where $\left.v_{h}\right|_{T^{\prime}} \in \Pi_{p}\left(T^{\prime}\right), T^{\prime} \in \mathcal{T}_{h}(\Omega)$, is uniquely determined by the degrees of freedom

$$
\begin{aligned}
& \int_{E} v_{h} q_{E} d s, \quad q_{E} \in \Pi_{p-1}(E), E \in \mathcal{E}_{h}\left(T^{\prime}\right), \\
& \int_{T} v_{h} q_{T^{\prime}} d x, \quad q_{T^{\prime}} \in \Pi_{p-3}\left(T^{\prime}\right) .
\end{aligned}
$$

For $i=1,2$, we choose $\varphi_{h} \in V_{h}$ with $\left.\varphi_{h}\right|_{T_{i}} \in \Pi_{p}\left(T_{i}\right)$ and $\varphi_{h} \mid T^{\prime} \equiv 0, T^{\prime} \in \mathcal{T}_{h}(\Omega) \backslash\{T\}$, such that

$$
\begin{aligned}
h_{T_{i}}^{2}\left\|\hat{f}_{T}+\Delta u_{H}\right\|_{T_{i}}^{2} & =\left(\hat{f}_{T}+\Delta u_{H}, \varphi_{h}\right)_{T_{i}}, \\
\left\|\varphi_{h}\right\|_{T_{i}}^{2} & \lesssim h_{T_{i}}^{4}\left\|\hat{f}_{T}+\Delta u_{H}\right\|_{T_{i}}^{2}, \\
\left(q_{h}, \varphi_{h}\right)_{E} & =0, \quad q_{h} \in \Pi_{p-1}(E), \quad E \in \mathcal{E}_{h}(\partial T) .
\end{aligned}
$$


In particular, in case $p \leq 2$ we choose $\varphi_{h}$ as a linear combination of the basis functions associated with the interior edge $E \in \mathcal{E}_{h}(\operatorname{int}(T))$, whereas for $p \geq 3$ we choose $\varphi_{h}$ as a linear combination of the basis functions associated with $\operatorname{int}\left(T_{i}\right)$. Using (4.4a), Green's formula, we obtain

$$
\begin{aligned}
h_{T}^{2}\left\|\hat{f}_{T}+\Delta u_{H}\right\|_{T}^{2}= & \sum_{i=1}^{2}\left(\hat{f}_{T}+\Delta u_{H}, \varphi_{h}\right)_{T_{i}} \\
& =\sum_{i=1}^{2}\left(-\left(\nabla u_{H}, \nabla \varphi_{h}\right)_{T_{i}}+\left(f, \varphi_{h}\right)_{T_{i}}+\left(\hat{f}_{T}-f, \varphi_{h}\right)_{T_{i}}\right),
\end{aligned}
$$

where we have used that

$$
\begin{aligned}
& \left(\partial_{n_{E}} u_{H}, \varphi_{h}\right)_{E}=0, \quad E \in \mathcal{E}_{h}(\partial T), \quad p \geq 1, \\
& \left(\partial_{n_{E}} u_{H},\left[\varphi_{h}\right]\right)_{E}=0, \quad E \in \mathcal{E}_{h}(\operatorname{int}(T)), \quad p \geq 1 .
\end{aligned}
$$

On the other hand, $\varphi_{h}$ is an admissible test function in the fine grid equation (2.4) whence

$$
\begin{aligned}
& \sum_{i=1}^{2}\left(\left(\nabla u_{h}, \nabla \varphi_{h}\right)_{T_{i}}-\left(f, \varphi_{h}\right)_{T_{i}}\right) \\
+ & \sum_{E \in \mathcal{E}_{h}\left(\partial T \cap \Gamma_{D}\right)}\left(u^{D}, \partial_{n_{E}} \varphi_{h}-\frac{\alpha}{h_{E}} \varphi_{h}\right)_{E} \\
- & \sum_{E \in \mathcal{E}_{h}\left(\partial T \cap \Gamma_{N}\right)}\left(u^{N}, \varphi_{h}\right)_{E} \\
- & \sum_{E \in \mathcal{E}_{h}(T)}\left(\left(\partial_{n_{E}}\left\{u_{h}\right\},\left[\varphi_{h}\right]\right)_{E}+\left(\left[u_{h}\right], \partial_{n_{E}}\left\{\varphi_{h}\right\}\right)_{E}\right) \\
+ & \sum_{E \in \mathcal{E}_{h}(T)} \frac{\alpha}{h_{E}}\left(\left[u_{h}\right],\left[\varphi_{h}\right]\right)_{E}=0 .
\end{aligned}
$$

Adding (4.5) and (4.7) and observing again (4.6a)-(4.6b) as well as $\left[u_{H}\right]=0$ on 
$E \in \mathcal{E}_{h}(\operatorname{int}(T))$, it follows that

$$
\begin{aligned}
h_{T}^{2}\left\|\hat{f}_{T}+\Delta u_{H}\right\|_{T}^{2}= & \sum_{i=1}^{2}\left(\left(\nabla\left(u_{h}-u_{H}\right), \nabla \varphi_{h}\right)_{T_{i}}+\left(\hat{f}_{T}-f, \varphi_{h}\right)_{T_{i}}\right) \\
& -\sum_{E \in \mathcal{E}_{h}(T)}\left(\left(\partial_{n_{E}}\left\{u_{h}-u_{H}\right\},\left[\varphi_{h}\right]\right)_{E}\right. \\
& \left.+\left(\left[u_{h}-u_{H}\right], \partial_{n_{E}}\left\{\varphi_{h}\right\}\right)_{E}\right) \\
& -\sum_{E \in \mathcal{E}_{h}(\partial T \cap \Omega)}\left(\left[u_{H}\right], \partial_{n_{E}}\left\{\varphi_{h}\right\}\right)_{E} \\
& +\sum_{E \in \mathcal{E}_{h}\left(\partial T \cap \Gamma_{D}\right)}\left(u^{D}-u_{H}, \partial_{n_{E}} \varphi_{h}-\frac{\alpha}{h_{E}} \varphi_{h}\right)_{E} \\
& -\sum_{E \in \mathcal{E}_{h}\left(\partial T \cap \Gamma_{N}\right)}\left(u^{N}-\hat{u}_{E}^{N}, \varphi_{h}\right)_{E} \\
& +\sum_{E \in \mathcal{E}_{h}(T)} \frac{\alpha}{h_{E}}\left(\left[u_{h}-u_{H}\right],\left[\varphi_{h}\right]\right)_{E} \\
& +\sum_{E \in \mathcal{E}_{h}\left(\partial T \backslash \Gamma_{D}\right)} \frac{\alpha}{h_{E}}\left(\left[u_{H}\right],\left[\varphi_{h}\right]\right)_{E} .
\end{aligned}
$$

In view of $(4.4 \mathrm{~b})$, the inverse inequality and the trace inequalities imply that

$$
\begin{aligned}
\left\|\nabla \varphi_{h}\right\|_{T_{i}}^{2} & \lesssim h_{T_{i}}^{2}\left\|\hat{f}_{T}+\Delta u_{H}\right\|_{T_{i}}^{2}, & & \\
\left\|\left[\varphi_{h}\right]\right\|_{E}^{2} & \lesssim h_{E}^{3}\left\|\hat{f}_{T}+\Delta u_{H}\right\|_{T_{i}}^{2}, & & E \in \mathcal{E}_{h}\left(\partial T_{i}\right), \\
\left\|\partial_{n_{E}}\left\{\varphi_{h}\right\}\right\|_{E}^{2} & \lesssim h_{E}\left\|\hat{f}_{T}+\Delta u_{H}\right\|_{T_{i}}^{2}, & & E \in \mathcal{E}_{h}\left(\partial T_{i}\right) .
\end{aligned}
$$

Then, using (4.4b) and (4.9a)-(4.9c) straightforward estimation of the terms on the right-hand side in (4.8) gives the assertion. $\square$

Lemma 4.4. Let $E \in \mathcal{E}_{H}(\Omega), E=T_{+} \cap T_{-}, T_{ \pm} \in \mathcal{T}_{H}(\Omega)$, be a refined edge and $\omega_{E}:=T_{+} \cup T_{-}$. Then, there holds

$$
\begin{aligned}
h_{E}\left\|\partial_{n_{E}}\left[u_{H}\right]\right\|_{E}^{2} \lesssim & \sum_{T_{ \pm} \in \mathcal{T}_{H}\left(\omega_{E}\right)} \eta_{T_{ \pm}}^{2}+\sum_{E^{\prime} \in \mathcal{E}_{H}\left(\omega_{E} \cap \Omega\right)} \eta_{E^{\prime}, 2}^{2} \\
& +\sum_{E^{\prime} \in \mathcal{E}_{H}\left(\partial \omega_{E} \cap \Gamma_{D}\right)} \eta_{E^{\prime}, D}^{2}+\sum_{E^{\prime} \in \mathcal{E}_{H}\left(\partial \omega_{E} \cap \Gamma_{N}\right)} \operatorname{osc}_{E^{\prime}}^{2}\left(u^{N}\right) .
\end{aligned}
$$

For a refined edge $E \in \mathcal{E}_{H}\left(\Gamma_{N}\right)$ with $E=\partial T \cap \Gamma_{N}, T \in \mathcal{T}_{H}(\Omega)$ we have

$$
h_{E}\left\|u^{N}-\partial_{n_{E}} u_{H}\right\|_{E}^{2} \lesssim \eta_{T}^{2}+\sum_{E^{\prime} \in \mathcal{E}_{H}(T \cap \Omega)} \eta_{E^{\prime}, 2}^{2} .
$$

Proof. For the proof of (4.10) let us assume that $E=T_{+} \cap T_{-}, T_{ \pm} \in \mathcal{T}_{H}(\Omega)$. We choose $\varphi_{H} \in C R_{p}\left(\Omega ; \mathcal{T}_{H}(\Omega)\right)$ as a linear combination of the basis functions associated with the edge $E$ such that

$$
\begin{aligned}
h_{E}\left\|\partial_{n_{E}}\left[u_{H}\right]\right\|_{E}^{2} & =\left(\partial_{n_{E}}\left[u_{H}\right], \varphi_{H}\right)_{E}, \\
\left\|\varphi_{H}\right\|_{T_{ \pm}} & \lesssim h_{E}^{3 / 2}\left\|\partial_{n_{E}}\left[u_{H}\right]\right\|_{E}, \\
\left(q_{E^{\prime}}, \varphi_{H}\right)_{E^{\prime}} & =0, \quad q_{E^{\prime}} \in \Pi_{p-1}\left(E^{\prime}\right),
\end{aligned}
$$


for any edge $E^{\prime}$. Using the definition of $\partial_{n_{E}}\left[u_{H}\right]$, it follows that

$$
\begin{aligned}
& \left(\partial_{n_{E}}\left[u_{H}\right], \varphi_{H}\right)_{E}= \\
= & \left(\nu_{E}^{+} \cdot \nabla u_{H}^{+}, \varphi_{H}\right)_{E}+\left(\nu_{E}^{-} \cdot \nabla u_{H}^{-}, \varphi_{H}\right)_{E} .
\end{aligned}
$$

By Green's formula, we find

$$
\begin{aligned}
& \left(\nabla u_{H}, \nabla \varphi_{H}\right)_{T_{ \pm}}= \\
& =-\left(\Delta u_{H}, \varphi_{H}\right)_{T_{ \pm}}+\sum_{E^{\prime} \in \mathcal{E}_{H}\left(\partial T_{ \pm}\right)}\left(\partial_{n_{E^{\prime}}} u_{H}, \varphi_{H}\right)_{E^{\prime}} .
\end{aligned}
$$

By (4.12c) we have

$$
\left(\partial_{n_{E^{\prime}}} u_{H}, \varphi_{H}\right)_{E^{\prime}}=0 \quad, \quad E^{\prime} \in \mathcal{E}_{H}\left(\partial \omega_{E}\right)
$$

whence

$$
\begin{gathered}
h_{E}\left\|\partial_{n_{E}}\left[u_{H}\right]\right\|_{E}^{2}= \\
=\left(\partial_{n_{E}}\left[u_{H}\right], \varphi_{H}\right)_{E}=\left(\nabla u_{H}, \nabla \varphi_{H}\right)_{\omega_{E}}+\left(\Delta u_{H}, \varphi_{H}\right)_{\omega_{E}} .
\end{gathered}
$$

On the other hand, since $\varphi_{H}$ is an admissible test function in (2.4), we have

$$
\begin{aligned}
&\left(\nabla u_{H}, \nabla \varphi_{H}\right)_{\omega_{E}}=\left(f, \varphi_{H}\right)_{\omega_{E}}+\sum_{E^{\prime} \in \mathcal{E}_{H}\left(\partial \omega_{E} \cap \Gamma_{N}\right)}\left(u^{N}, \varphi_{H}\right)_{E^{\prime}}- \\
&-\sum_{E^{\prime} \in \mathcal{E}_{H}\left(\partial \omega_{E} \cap \Gamma_{D}\right)}\left(u^{D}, \partial_{n_{E^{\prime}}} \varphi_{H}-\frac{\alpha}{h_{E}^{\prime}} \varphi_{H}\right)_{E^{\prime}}+ \\
&+\sum_{E^{\prime} \in \mathcal{E}_{H}\left(\omega_{E}\right)}\left(\left[u_{H}\right], \partial_{n_{E^{\prime}}}\left\{\varphi_{H}\right\}\right)_{E^{\prime}}-\sum_{E^{\prime} \in \mathcal{E}_{H}\left(\partial \omega_{E}\right)} \frac{\alpha}{h_{E}^{\prime}}\left(\left[u_{H}\right],\left[\varphi_{H}\right]\right)_{E^{\prime}}
\end{aligned}
$$

where we have used (4.15) and (4.12c) on E. Combining (4.16) and (4.17) results in

$$
\begin{aligned}
h_{E}\left\|\partial_{n_{E}}\left[u_{H}\right]\right\|_{E}^{2}= & \left(f+\Delta u_{H}, \varphi_{H}\right)_{\omega_{E}} \\
& +\sum_{E^{\prime} \in \mathcal{E}_{H}\left(\omega_{E} \backslash \Gamma_{D}\right)}\left(\left[u_{H}\right], \partial_{n_{E^{\prime}}}\left\{\varphi_{H}\right\}\right)_{E^{\prime}} \\
& -\sum_{E^{\prime} \in \mathcal{E}_{H}\left(\partial \omega_{E} \backslash \Gamma_{D}\right)} \frac{\alpha}{h_{E}^{\prime}}\left(\left[u_{H}\right],\left[\varphi_{H}\right]\right)_{E^{\prime}} \\
& +\sum_{E^{\prime} \in \mathcal{E}_{H}\left(\partial \omega_{E} \cap \Gamma_{N}\right)}\left(u^{N}-\hat{u}_{E^{\prime}}^{N}, \varphi_{H}\right)_{E^{\prime}} \\
& -\sum_{E^{\prime} \in \mathcal{E}_{H}\left(\partial \omega_{E} \cap \Gamma_{D}\right)}\left(u^{D}-u_{H}, \partial_{n_{E^{\prime}}} \varphi_{H}-\frac{\alpha}{h_{E}^{\prime}} \varphi_{H}\right)_{E^{\prime}} .
\end{aligned}
$$

Observing (4.12b), the trace inequalities yield

$$
\begin{aligned}
\left\|\left[\varphi_{H}\right]\right\|_{E^{\prime}} & \lesssim h_{E}\left\|\partial_{n_{E}}\left[u_{H}\right]\right\|_{E}, & & E^{\prime} \in \mathcal{E}_{H}\left(\partial \omega_{E}\right), \\
\left\|\partial_{n_{E^{\prime}}}\left\{\varphi_{H}\right\}\right\|_{E^{\prime}} & \lesssim\left\|\partial_{n_{E}}\left[u_{H}\right]\right\|_{E}, & & E^{\prime} \in \mathcal{E}_{H}\left(\partial \omega_{E}\right) .
\end{aligned}
$$

Taking advantage of (4.12b),(4.19a) and (4.19b), the assertion can be deduced by straightforward estimation of the terms on the right-hand side in (4.18). The proof of (4.11) follows by similar arguments. 
Lemma 4.5. Let $E \in \mathcal{E}_{H}(\Omega), E=T_{+} \cap T_{-}, T_{ \pm} \in \mathcal{T}_{H}(\Omega)$, be a refined edge and $\omega_{E}:=T_{+} \cup T_{-}$. Then, there holds

$$
\left.\frac{\alpha}{h_{E}}\left\|\left.\left[u_{H}\right]\right|_{E}\right\|_{E}^{2} \lesssim a_{h}\right|_{\omega_{E}}\left(u_{h}-u_{H}, u_{h}-u_{H}\right)+\sum_{E^{\prime} \in \mathcal{E}_{h}(E)} \frac{\alpha}{h_{E}^{\prime}}\left\|\left[u_{h}\right]\right\|_{E^{\prime}}^{2}
$$

Likewise, if $E \in \mathcal{E}_{H}\left(\Gamma_{D}\right)$ is a refined edge such that $E=\partial T \cap \Gamma_{D}, T \in \mathcal{T}_{H}(\Omega)$, there holds

$$
\begin{aligned}
\left.\frac{\alpha}{h_{E}}\left\|u^{D}-u_{H}\right\|_{E}^{2} \lesssim a_{h}\right|_{T}\left(u_{h}-u_{H}, u_{h}-u_{H}\right) & \\
& +\sum_{E^{\prime} \in \mathcal{E}_{h}(E)} \frac{\alpha}{h_{E}^{\prime}}\left\|u^{D}-u_{h}\right\|_{E^{\prime}}^{2}+\operatorname{osc}_{E}^{2}\left(u^{D}\right) .
\end{aligned}
$$

Proof. For the proof of $(4.20)$ choose $\psi_{H}^{ \pm} \in C R_{p}\left(\Omega ; \mathcal{T}_{H}(\Omega)\right)$ with $\operatorname{supp}\left(\psi_{H}^{ \pm}\right)=T_{ \pm}$ as a linear combination of basis functions associated with $E$ such that

$$
\begin{aligned}
\left(\left[u_{H}\right], \psi_{H}^{ \pm}\right)_{E} & = \pm \frac{1}{2}\left\|\left[u_{H}\right]\right\|_{E}^{2}, \\
\left\|\psi_{H}^{ \pm}\right\|_{T_{ \pm}} & \lesssim h_{E}^{1 / 2}\left\|\left[u_{H}\right]\right\|_{E} .
\end{aligned}
$$

We define $\varphi_{H} \in V_{H}$ by $\left.\varphi_{H}\right|_{T_{ \pm}}=\psi_{H}^{ \pm}$and $\left.\varphi_{H}\right|_{T} \equiv 0, T \in \mathcal{T}_{H}(\Omega) \backslash\left\{\omega_{E}\right\}$. Then, it follows from (4.22a) that

$$
\frac{\alpha}{h_{E}}\left\|\left.\left[u_{H}\right]\right|_{E}\right\|_{E}^{2}=\frac{\alpha}{h_{E}}\left(\left[u_{H}\right],\left[\varphi_{H}\right]\right)_{E} .
$$

Since $\varphi_{H}$ is an admissible test function in (2.4), we have

$$
\begin{aligned}
\frac{\alpha}{h_{E}}\left(\left[u_{H}\right],\left[\varphi_{H}\right]\right)_{E}= & \left(f, \varphi_{H}\right)_{\omega_{E}}-\left(\nabla u_{H}, \nabla \varphi_{H}\right)_{\omega_{E}} \\
& +\sum_{E^{\prime} \in \mathcal{E}_{H}\left(\partial \omega_{E} \cup\{E\}\right)}\left(\partial_{n_{E^{\prime}}}\left\{u_{H}\right\},\left[\varphi_{H}\right]\right)_{E^{\prime}} \\
& +\sum_{E^{\prime} \in \mathcal{E}_{H}\left(\partial \omega_{E}\right) \cup\{E\}}\left(\left[u_{H}\right], \partial_{n_{E^{\prime}}}\left\{\varphi_{H}\right\}\right)_{E^{\prime}} \\
& -\sum_{E^{\prime} \in \mathcal{E}_{H}\left(\partial \omega_{E}\right)} \frac{\alpha}{h_{E}^{\prime}}\left(\left[u_{H}\right],\left[\varphi_{H}\right]\right)_{E^{\prime}} \\
& +\sum_{E^{\prime} \in \mathcal{E}_{H}\left(\partial \omega_{E} \cap \Gamma_{N}\right)}\left(u^{N}, \varphi_{H}\right)_{E^{\prime}} \\
& -\sum_{E^{\prime} \in \mathcal{E}_{H}\left(\partial \omega_{E} \cap \Gamma_{D}\right)}\left(u^{D}, \partial_{n_{E^{\prime}}} \varphi_{H}-\frac{\alpha}{h_{E}^{\prime}} \varphi_{H}\right)_{E^{\prime}} .
\end{aligned}
$$

On the other hand, $\left(\left.\varphi_{H}\right|_{T^{\prime}}\right)_{T^{\prime} \in \mathcal{T}_{h}(\Omega)}$ is an admissible test function in the fine grid 
equation (2.4). Hence, observing $\left[\varphi_{H}\right]=0$ and $\left[u_{H}\right]=0$ on $E^{\prime} \in \mathcal{E}_{h}\left(\operatorname{int}\left(T_{ \pm}\right)\right)$, we get

$$
\begin{aligned}
0= & \left(\nabla u_{h}, \nabla \varphi_{H}\right)_{\omega_{E}}-\left(f, \varphi_{H}\right)_{\omega_{E}} \\
& -\sum_{E^{\prime} \in \mathcal{E}_{h}\left(\partial \omega_{E} \cup\{E\}\right)}\left(\partial_{n_{E^{\prime}}}\left\{u_{h}\right\},\left[\varphi_{H}\right]\right)_{E^{\prime}} \\
& -\sum_{E^{\prime} \in \mathcal{E}_{h}\left(\partial \omega_{E} \cup\{E\}\right)}\left(\left[u_{h}\right], \partial_{n_{E^{\prime}}}\left\{\varphi_{H}\right\}\right)_{E^{\prime}} \\
& -\sum_{E^{\prime} \in \mathcal{E}_{h}\left(i n t\left(\omega_{E}\right) \backslash\{E\}\right)}\left(\left[u_{h}-u_{H}\right], \partial_{n_{E^{\prime}}}\left\{\varphi_{H}\right\}\right)_{E^{\prime}} \\
& +\sum_{E^{\prime} \in \mathcal{E}_{h}\left(\partial \omega_{E} \cup\{E\}\right)} \frac{\alpha}{h_{E}^{\prime}}\left(\left[u_{h}\right],\left[\varphi_{H}\right]\right)_{E^{\prime}} \\
& -\sum_{E^{\prime} \in \mathcal{E}_{h}\left(\partial \omega_{E} \cap \Gamma_{N}\right)}\left(u^{N}, \varphi_{H}\right)_{E^{\prime}} \\
& +\sum_{E^{\prime} \in \mathcal{E}_{h}\left(\partial \omega_{E} \cap \Gamma_{D}\right)}\left(u^{D}, \partial_{n_{E^{\prime}}} \varphi_{H}-\frac{\alpha}{h_{E}^{\prime}} \varphi_{H}\right)_{E^{\prime}} .
\end{aligned}
$$

In view of $(4.22 \mathrm{~b})$, the inverse inequality and the trace inequalities imply

$$
\begin{aligned}
\left\|\nabla \psi_{H}^{ \pm}\right\|_{T_{ \pm}} & \lesssim h_{E}^{-1 / 2}\left\|\left[u_{H}\right]\right\|_{E}, & & \\
\left\|\psi_{H}^{ \pm}\right\|_{E^{\prime}} & \lesssim\left\|\left[u_{H}\right]\right\|_{E}, & & E^{\prime} \in \mathcal{E}_{H}\left(\omega_{E}\right), \\
\left\|\partial_{n_{E^{\prime}}} \psi_{H}^{ \pm}\right\|_{E^{\prime}} & \lesssim h_{E}^{-1}\left\|\left[u_{H}\right]\right\|_{E}, & & E^{\prime} \in \mathcal{E}_{H}\left(\omega_{E}\right) .
\end{aligned}
$$

Combining (4.24),(4.25) and using (4.22b),(4.26a)-(4.26c), straightforward estimation gives the assertion.

The proof of (4.21) can be established similarly.

5. Proof of the error reduction property. In the convergence analysis of standard finite element methods $[18,31]$, the proof of the error reduction property makes essential use of Galerkin orthogonality which in the framework of IPDG reads as follows

$$
a_{h}\left(u_{h}-u_{H}, u_{h}-u_{H}\right)=a_{h}\left(e_{H}, e_{H}\right)-a_{h}\left(e_{h}, e_{h}\right) .
$$

However, we measure the error $e_{H}$ with respect to the mesh dependent energy norm $a_{H}(\cdot, \cdot)$ associated with the coarse mesh $\mathcal{T}_{H}(\Omega)$ and hence, (5.1) can not be used directly. It is known from the convergence analysis of adaptive nonconforming finite elements [10] or of mixed finite elements [11] that in the absence of Galerkin orthogonality convergence can be established provided some sort of perturbed Galerkin orthogonality holds true. For the IPDG under consideration, we can rewrite (5.1) according to

$$
a_{h}\left(u_{h}-u_{H}, u_{h}-u_{H}\right)=\left(1+\delta_{h, H}\left(e_{H}\right)\right) a_{H}\left(e_{H}, e_{H}\right)-a_{h}\left(e_{h}, e_{h}\right),
$$

where in case $a_{H}\left(e_{H}, e_{H}\right) \neq 0$ the perturbation term $\delta_{h, H}\left(e_{H}\right)$ is given by

$$
\delta_{h, H}\left(e_{H}\right):=\frac{a_{h}\left(e_{H}, e_{H}\right)-a_{H}\left(e_{H}, e_{H}\right)}{a_{H}\left(e_{H}, e_{H}\right)} .
$$


We would be able to conclude, if we can show that $\delta_{h, H}\left(e_{H}\right)$ can be made sufficiently small.

Lemma 5.1 (Perturbed Galerkin orthogonality). There exists a positive constant $C_{1}$ depending only on the local geometry of the triangulations such that for the perturbation term $\delta_{h, H}\left(e_{H}\right)$ there holds

$$
\delta_{h, H}\left(e_{H}\right) \leq \frac{C_{1}}{\alpha}
$$

Proof. From (2.3), it is obvious (see also (3.8) in [21]) that

$$
a_{h}\left(e_{H}, e_{H}\right) \leq a_{H}\left(e_{H}, e_{H}\right)+\sum_{E \in \mathcal{E}_{H}(\Omega)} \frac{\alpha}{h_{E}}\left\|\left[u_{H}\right]\right\|_{E}^{2}+\sum_{E \in \mathcal{E}_{H}\left(\Gamma_{D}\right)} \frac{\alpha}{h_{E}}\left\|u^{D}-u_{H}\right\|_{E}^{2} .
$$

On the other hand, the local efficiency of the residual estimator (cf. [28]) tells us that there exists a positive constant $C_{1}$ depending only on the local geometry of the triangulations such that

$$
\sum_{E \in \mathcal{E}_{H}(\Omega)} \frac{\alpha}{h_{E}}\left\|\left[u_{H}\right]\right\|_{E}^{2}+\sum_{E \in \mathcal{E}_{H}\left(\Gamma_{D}\right)} \frac{\alpha}{h_{E}}\left\|u^{D}-u_{H}\right\|_{E}^{2} \leq \frac{C_{1}}{\alpha} a_{H}\left(e_{H}, e_{H}\right) .
$$

Combining the two preceding estimates allows to conclude.

Proof of Theorem 3.1. The reliability, the bulk criterion, and the discrete local efficiency infer the existence of a positive constant $C_{2}$ depending only on $\gamma, \Theta$ and the local geometry of the triangulations such that

$$
\begin{aligned}
a_{H}\left(e_{H}, e_{H}\right) \leq C_{2}\left(a _ { h } \left(u_{h}-u_{H},\right.\right. & \left.u_{h}-u_{H}\right)+\operatorname{osc}_{H}^{2} \\
& \left.+\sum_{E \in \mathcal{E}_{h}(\Omega)} \frac{\alpha}{h_{E}}\left\|\left[u_{h}\right]\right\|_{E}^{2}+\sum_{E \in \mathcal{E}_{h}\left(\Gamma_{D}\right)} \frac{\alpha}{h_{E}}\left\|u_{D}-u_{h}\right\|_{E}^{2}\right) .
\end{aligned}
$$

Using (5.2),(5.4) and (5.5) with $h$ instead of $H$, we obtain the existence of a positive constant $C_{3}$ such that

$$
a_{H}\left(e_{H}, e_{H}\right) \leq C_{2}\left(1+\frac{C_{1}}{\alpha}\right) a_{H}\left(e_{H}, e_{H}\right)-\left(C_{2}-\frac{C_{3}}{\alpha}\right) a_{h}\left(e_{h}, e_{h}\right)+C_{2} \operatorname{osc}_{H}^{2},
$$

from which we deduce

$$
a_{h}\left(e_{h}, e_{h}\right) \leq\left(C_{2}-\frac{C_{3}}{\alpha}\right)^{-1}\left[\left(\left(1+\frac{C_{1}}{\alpha}\right) C_{2}-1\right) a_{H}\left(e_{H}, e_{H}\right)+C_{2} \operatorname{osc}_{H}^{2}\right] .
$$

For $\alpha>C_{1} C_{2}+C_{3}$, the error reduction property (3.10) results with

$$
\rho_{1}:=\left(C_{2}-\frac{C_{3}}{\alpha}\right)^{-1}\left(\left(1+\frac{C_{1}}{\alpha}\right) C_{2}-1\right)<1 .
$$


TABLE 6.1 and 4

Decline of energy norm and data oscillation in terms of refinement step, polynomial degrees 1

\begin{tabular}{|c|ccc|ccc|}
\hline & \multicolumn{3}{|c|}{$P_{1}$} & \multicolumn{3}{c|}{$P_{4}$} \\
\hline$l$ & $N_{\text {dof }}$ & $\left\|e_{l}\right\|_{A}$ & osc $_{l}$ & $N_{\text {dof }}$ & $\left\|e_{l}\right\|_{A}$ & osc $_{l}$ \\
\hline 0 & 36 & $2.81 \mathrm{e}-1$ & $9.32 \mathrm{e}-2$ & 180 & $6.07 \mathrm{e}-2$ & $9.32 \mathrm{e}-2$ \\
1 & 114 & $1.98 \mathrm{e}-1$ & $6.83 \mathrm{e}-2$ & 570 & $3.83 \mathrm{e}-2$ & $6.83 \mathrm{e}-2$ \\
2 & 252 & $1.39 \mathrm{e}-1$ & $3.35 \mathrm{e}-2$ & 960 & $2.60 \mathrm{e}-2$ & $5.38 \mathrm{e}-2$ \\
3 & 630 & $9.53 \mathrm{e}-2$ & $1.66 \mathrm{e}-2$ & 1440 & $1.75 \mathrm{e}-2$ & $3.35 \mathrm{e}-2$ \\
4 & 1428 & $6.48 \mathrm{e}-2$ & $1.07 \mathrm{e}-2$ & 2280 & $1.23 \mathrm{e}-2$ & $2.28 \mathrm{e}-2$ \\
5 & 3180 & $4.42 \mathrm{e}-2$ & $5.85 \mathrm{e}-3$ & 3000 & $7.72 \mathrm{e}-3$ & $1.71 \mathrm{e}-2$ \\
6 & 6714 & $3.00 \mathrm{e}-2$ & $4.10 \mathrm{e}-3$ & 4020 & $4.86 \mathrm{e}-3$ & $1.15 \mathrm{e}-2$ \\
7 & 14076 & $2.08 \mathrm{e}-2$ & $2.46 \mathrm{e}-3$ & 5355 & $3.06 \mathrm{e}-3$ & $8.41 \mathrm{e}-3$ \\
8 & 28368 & $1.43 \mathrm{e}-2$ & $1.51 \mathrm{e}-3$ & 6330 & $1.93 \mathrm{e}-3$ & $6.20 \mathrm{e}-3$ \\
9 & 58461 & $9.91 \mathrm{e}-3$ & $9.18 \mathrm{e}-4$ & 7620 & $1.22 \mathrm{e}-3$ & $4.31 \mathrm{e}-3$ \\
\hline
\end{tabular}

6. Computational results. In the following numerical experiments we used the bisection algorithm, for all test cases, derived from the AFEM@Matlab implementation [14].

We verify the suitability of our theoretical results using standard test cases (see [10]). They are studies of the behavior of the algorithm in case of the standard singularities induced by a reentrant corner of the domain. The right hand side is chosen to zero and therefore, data oscillations are only present on the the boundary edges not adjacent to the singularity, where values of the analytical solutions are prescribed. First, we study the L-shaped domain with Dirichlet data $u^{D}=0$ on the two edges adjacent to the reentrant corner and Neumann data on the remaining boundary. The refinement parameter is chosen as $\Theta=0.6$. Table 6.1 shows a decline of the energy norm for this case by a factor of about $2 / 3$ in each refinement step. This factor is only slightly better for quartic shape functions, confirming that the reduction rate depends mostly on $\Theta$. Nevertheless, the meshes for $P_{4}$ are growing much slower and in both cases we obtain the optimal approximation rates in terms of $N_{\text {dof }}$, namely $N_{\text {dof }}^{-1 / 2}$ and $N_{\text {dof }}^{-2}$. Data oscillation occurs only at the outer boundary and is negligible in this case.

Next, we study the higher singularity of the slit domain. Here, Dirichlet boundary conditions are used on the whole boundary. Table 6.2 shows that for $\Theta=0.4$, we obtain again constant error reduction rates.

The solution in this example is highly singular and we expect that at least for higher order polynomials, the refinement should be very local. Indeed, Figure 6.1 shows that the parameter $\Theta$ must be chosen carefully in order to obtain optimal approximation with respect to the degrees of freedom, confirming results from [37] for standard AFEM. Only the very small value of $\Theta=0.1$ is able to reproduce the optimal convergence order of $N^{-2}$. Figure 6.2 shows, that this corresponds asymptotically to only adding $1 / 16$ th of the current number of cells in each step.

Even with the small size of $\Theta=0.1$, the optimal $N$-term approximation rate is only obtained after several thousand degrees of freedom. Comparing Figures 6.1 and 6.2 , we note that this corresponds to the fact that the bulk criterion refines much faster than the asymptotic rate in its initialization phase. On the other hand, this fast refinement allows the method to reach the asymptotic regime in only about 10 
TABLE 6.2

Decline of energy and data oscillation in terms of refinement step, polynomial degrees 1 and 4

\begin{tabular}{|c|ccc|ccc|}
\hline & \multicolumn{3}{|c|}{$P_{1}$} & \multicolumn{3}{c|}{$P_{4}$} \\
\hline$l$ & $N_{\text {dof }}$ & $\left\|e_{l}\right\|_{A}$ & osc $_{l}$ & $N_{\text {dof }}$ & $\left\|e_{l}\right\|_{A}$ & osc $_{l}$ \\
\hline 0 & 18 & $8.83 \mathrm{e}-1$ & $2.32 \mathrm{e}-1$ & 90 & $5.16 \mathrm{e}-1$ & $2.37 \mathrm{e}-1$ \\
1 & 48 & $6.90 \mathrm{e}-1$ & $2.09 \mathrm{e}-1$ & 165 & $4.39 \mathrm{e}-1$ & $2.20 \mathrm{e}-1$ \\
2 & 138 & $5.76 \mathrm{e}-1$ & $1.80 \mathrm{e}-1$ & 285 & $3.61 \mathrm{e}-1$ & $1.95 \mathrm{e}-1$ \\
3 & 219 & $4.86 \mathrm{e}-1$ & $1.61 \mathrm{e}-1$ & 690 & $3.03 \mathrm{e}-1$ & $1.83 \mathrm{e}-1$ \\
$\ldots$ & & & & & & \\
18 & 54804 & $4.42 \mathrm{e}-2$ & $3.59 \mathrm{e}-2$ & 32400 & $2.27 \mathrm{e}-2$ & $4.69 \mathrm{e}-2$ \\
19 & 76809 & $3.73 \mathrm{e}-2$ & $3.21 \mathrm{e}-2$ & 39630 & $1.91 \mathrm{e}-2$ & $4.22 \mathrm{e}-2$ \\
20 & 106821 & $3.16 \mathrm{e}-2$ & $2.88 \mathrm{e}-2$ & 52605 & $1.59 \mathrm{e}-2$ & $3.79 \mathrm{e}-2$ \\
21 & 149829 & $2.67 \mathrm{e}-2$ & $2.57 \mathrm{e}-2$ & 63480 & $1.34 \mathrm{e}-2$ & $3.41 \mathrm{e}-2$ \\
\hline
\end{tabular}

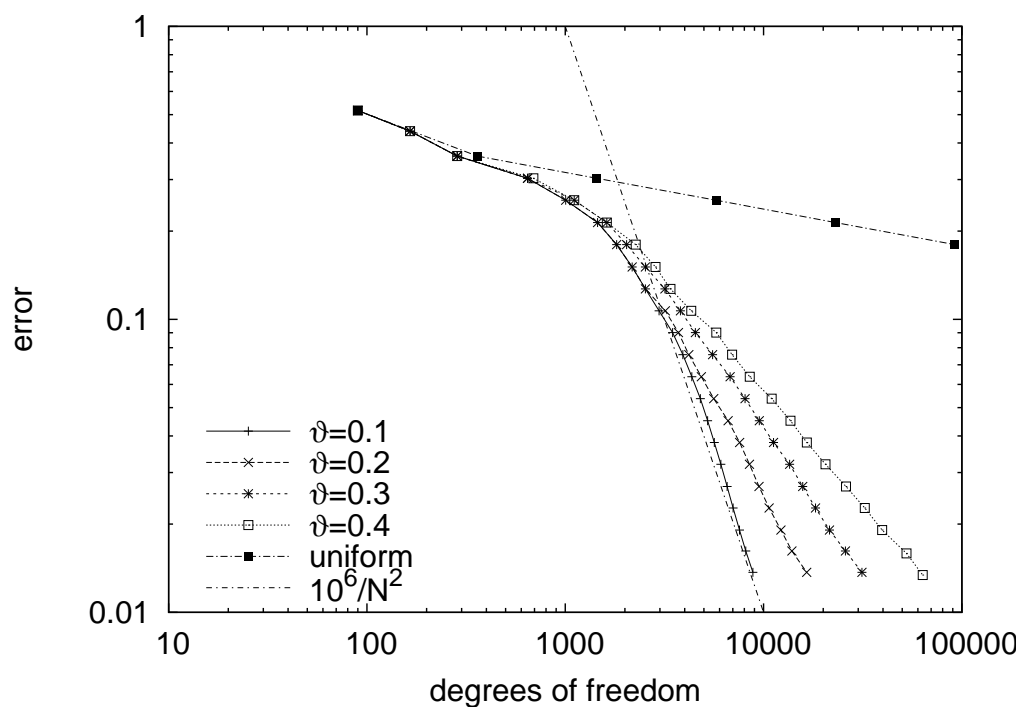

FIG. 6.1. Error versus number of degrees of freedom for the slit domain, quartic polynomials

steps. Figure 6.3 shows that the refinement for $\Theta=0.1$ is much more concentrated at the central singularity, while $\Theta=0.4$ puts more weight in reducing the boundary projection errors.

\section{REFERENCES}

[1] M. Ainsworth and J.T. Oden. A Posteriori Error Estimation in Finite Element Analysis. Wiley, Chichester, 2000.

[2] P. F. Antonietti and B. Ayuso, Schwarz domain decomposition preconditioners for discontinuous Galerkin approximations of elliptic problems: non-overlapping case. Math. Model. Numer. Anal. 41, no. 1, 21-54, 2007.

[3] D. N. Arnold. An interior penalty finite element method with discontinuous elements. SIAM J. Numer. Anal. 19, 742-760, 1982.

[4] D. N. Arnold, F. Brezzi, B. Cockburn, and D. Marini. Unified analysis of discontinuous Galerkin methods for elliptic problems. SIAM J. Numer. Anal. 39, 1749-1779, 2001.

[5] I. Babuska and T. Strouboulis. The Finite Element Method and its Reliability. Clarendon Press, 


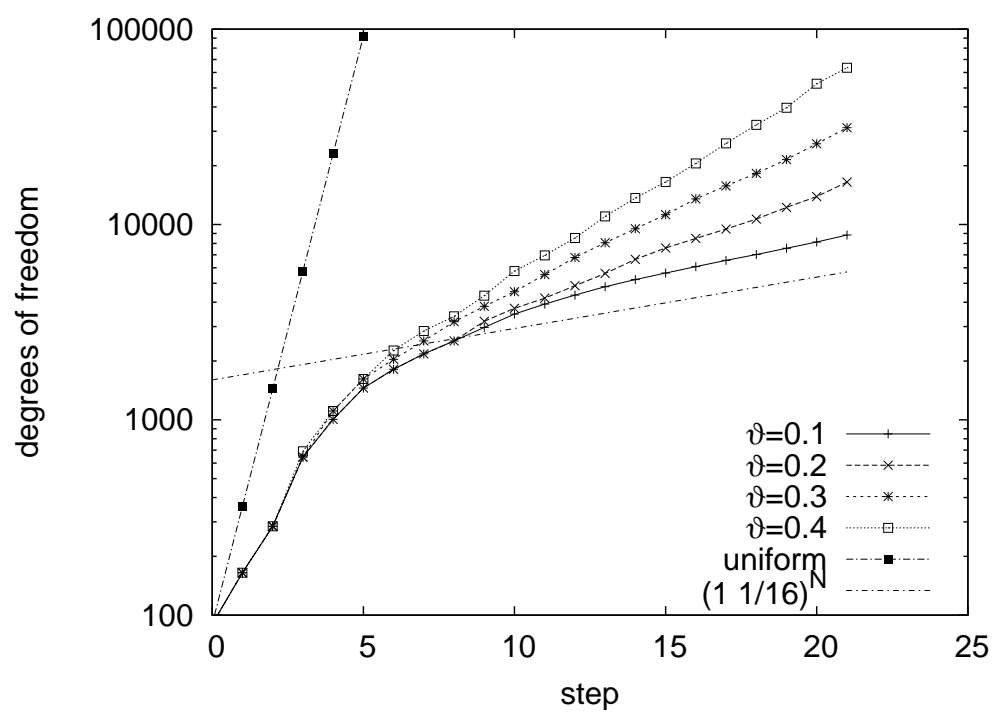

FIG. 6.2. Development of mesh sizes during adaptive refinement, quartic polynomials
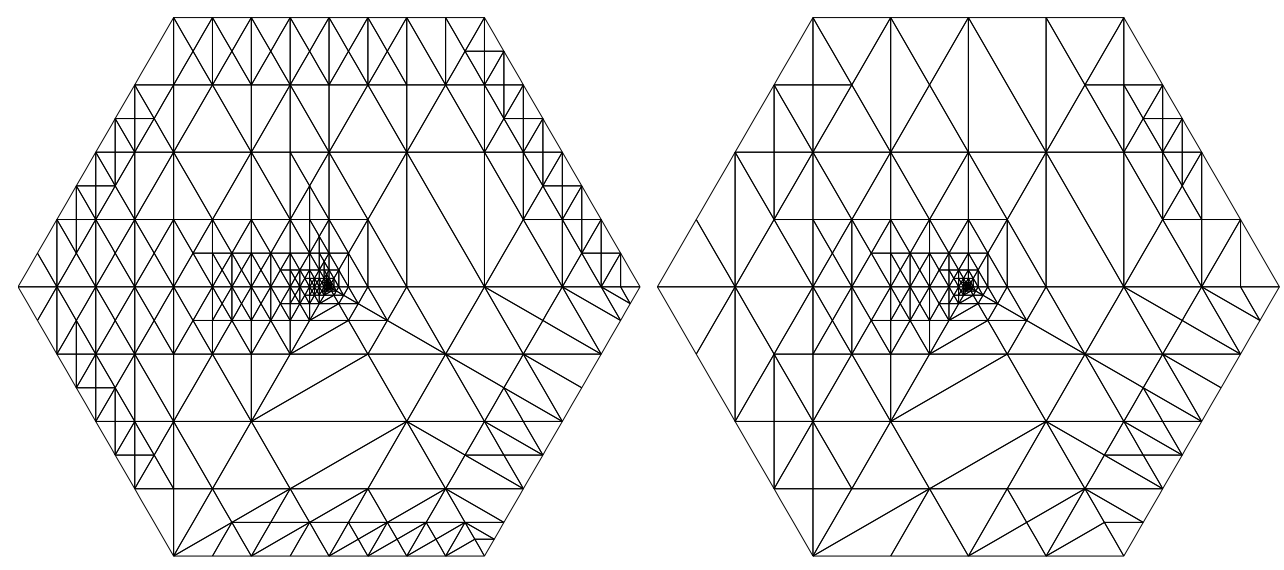

FiG. 6.3. Meshes for the slit domain, polynomial degree 4, $\Theta=0.4$ (left) and $\Theta=0.1$ (right).

Oxford, 2001.

[6] W. Bangerth and R. Rannacher. Adaptive Finite Element Methods for Differential Equations. Lectures in Mathematics. ETH-Zürich. Birkhäuser, Basel, 2003.

[7] R. Becker, P. Hansbo, and M.G. Larson. Energy norm a posteriori error estimation for discontinuous Galerkin methods. Comput. Methods Appl. Mech. Engrg. 192, 723-733, 2003.

[8] P. Binev, W. Dahmen, and R. DeVore. Adaptive Finite Element Methods with Convergence Rates. Numer. Math. 97, 219-268, 2004.

[9] C. Carstensen and R. H. W. Hoppe. Convergence analysis of an adaptive edge finite element method for the 2d eddy current equations. J. Numer. Math. 13, 19-32, 2005.

[10] C. Carstensen and R. H. W. Hoppe. Convergence analysis of an adaptive nonconforming finite element method. Numer. Math. 103, 251-266, 2006

[11] C. Carstensen and R. H. W. Hoppe. Error reduction and convergence for an adaptive mixed finite element method. Math. Comp. 75, 1033-1042, 2006

[12] P. Castillo, B. Cockburn, I. Perugia, and D. Schötzau. An a priori error estimate of the local discontinuous Galerkin method for elliptic problems. SIAM J. Numer. Anal. 38, 1676-1706, 2000. 
[13] L. Chen. Short bisection implementation in MATLAB. Preprint. Department of Mathematics, University of Maryland, 2006.

[14] L. Chen and C. Zhang. AFEM@matlab: a MATLAB package of adaptive finite element methods. Technical Report. University of Maryland. 2006.

[15] B. Cockburn. Discontinuous Galerkin methods. Z. Angew. Math. Mech. 83, 731-754, 2003.

[16] B. Cockburn, G.E. Karniadakis, and C.-W. Shu (eds.). Discontinuous Galerkin Methods. Lecture notes in Computational Science and Engineering, Vol. 11, Springer, Berlin-HeidelbergNew York, 2000.

[17] B. Cockburn, G. Kanschat, I. Perugia, and D. Schötzau. Superconvergence of the local discontinuous Galerkin method for elliptic problems on cartesian grids. SIAM J. Numer. Anal. 39, 264-285, 2001.

[18] W. Dörfler. A convergent adaptive algorithm for Poisson's equation. SIAM J. Numer. Anal., 33, 1106-1124, 1996.

[19] K. Eriksson, D. Estep, P. Hansbo, and C. Johnson. Computational Differential Equations. Cambridge University Press, Cambridge, 1995.

[20] J. Gopalakrishnan and G. Kanschat. Multi-level preconditioners for the interior penalty method. In: Numerical Mathematics and Advanced Applications, Proc. ENUMATH 2001 (F. Brezzi et al.; eds.), pp. 795-804, Springer, Milano, 2003.

[21] J. Gopalakrishnan and G. Kanschat. A multilevel discontinuous Galerkin method. Numer. Math. 95, 527-550, 2003.

[22] J. Guzmán. Pointwise error estimates for discontinuous Galerkin methods with lifting operators for elliptic problems. Math. Comput. 75, no. 255, 1067-1085, 2006.

[23] P. Houston, I. Perugia, and D. Schötzau. Energy norm a posteriori error estimation for mixed discontinuous Galerkin approximations of the Maxwell operator. Comput. Methods Appl. Mech. Engrg. 194, 499-510, 2005.

[24] P. Houston, I. Perugia, and D. Schötzau. A posteriori error estimation for discontinuous Galerkin discretizations of H(curl)-elliptic partial differential equations. to appear in IMA J. Numer. Anal.

[25] P. Houston, D. Schötzau, and T. Wihler. Energy norm a posteriori error estimation for mixed discontinuous Galerkin approximations of the Stokes problem. J. Sci. Comp. 22, 357-380, 2005.

[26] G. Kanschat. Discontinuous Galerkin Methods for Viscous Incompressible Flow. Deutscher Universitätsverlag, 2007.

[27] G. Kanschat and R. Rannacher. Local error analysis of the interior penalty discontinuous Galerkin method for second order problems. J. Numer. Math. 10, 249-274, 2002.

[28] O. Karakashian and F. Pascal. A posteriori error estimates for a discontinuous Galerkin approximation of second-order elliptic problems. SIAM J. Numer. Anal. 41, 2374-2399, 2003.

[29] O. Karakashian and F. Pascal. Convergence of adaptive discontinuous Galerkin approximations of second-order elliptic problems. Preprint. Department of Mathematics, University of Tennessee, 2007.

[30] K. Mekchay and R. Nochetto. Convergence of adaptive finite element methods for general second order linear elliptic PDE. SIAM J. Numer. Anal. 43, 1803-1827, 2005.

[31] P. Morin, R.H. Nochetto, and K.G. Siebert. Data Oscillation and convergence of adaptive FEM. SIAM J. Numer. Anal., 38, 466-488, 2000.

[32] P. Morin, R.H. Nochetto, and K.G. Siebert. Convergence of adaptive finite element methods. SIAM REVIEW 44 631-658, 2002.

[33] P. Neittaanmäki and S. Repin. Reliable methods for mathematical modelling. Error control and a posteriori estimates. Elsevier, New York, 2004.

[34] B. Rivière, M.F. Wheeler, and V. Girault. Improved energy estimates for interior penalty, constrained and discontinuous Galerkin methods for elliptic problems. Part I. Comp. Geo. 3, 337-360, 1999.

[35] B. Rivière and M.F. Wheeler. A posteriori error estimates and mesh adaptation strategy for discontinuous Galerkin methods applied to diffusion problems. Computers \& Mathematics with Applications 46, 141-163, 2003.

[36] A. Schmidt and K. G. Siebert. Design of adaptive finite element software: the finite element toolbox ALBERTA Lecture Notes in Computational Science and Engineering 42, SpringerVerlag, Berlin, 2005

[37] R. Stevenson. Optimality of a standard adaptive finite element method. Found. Comput. Math., DOI 10.1007/s 10208-005-0183-0, 2006.

[38] R. Verfürth. A Review of A Posteriori Estimation and Adaptive Mesh-Refinement Techniques. Wiley-Teubner, New York, Stuttgart, 1996. 